\title{
On certain hypergeometric identities deducible by using the beta integral method
}

Adel K Ibrahim ${ }^{1,3}$, Medhat A Rakha ${ }^{2,3^{*}}$ and Arjun K Rathie ${ }^{4}$

\section{${ }^{*}$ Correspondence:}

medhat@squ.edu.om

${ }^{2}$ Department of Mathematics and

Statistics - College of Science, Sultan

Qaboos University, Alkhoud, P.O. Box

36, Muscat, 123, Oman

${ }^{3}$ Permanent address: Department of Mathematics, Faculty of Science,

Suez Canal University, Ismailia,

Egypt

Full list of author information is

available at the end of the article

\begin{abstract}
The aim of this research paper is to demonstrate how one can obtain eleven new and interesting hypergeometric identities (in the form of a single result) from the old ones by mainly applying the well-known beta integral method which was used successfully and systematically by Krattenthaler and Rao in their well known, very interesting research papers. The results are derived with the help of generalization of a quadratic transformation formula due to Kummer very recently obtained by Kim et al. Several identities, including one obtained earlier by Krattenthaler and Rao, follow special cases of our main findings. The results established in this paper are simple, interesting, easily established and may be potentially useful.
\end{abstract}

MSC: Primary 33C05; 33C20; secondary 33C70

Keywords: hypergeometric series; Kummer summation theorem; beta integral

\section{Introduction and preliminaries}

The generalized hypergeometric series ${ }_{p} F_{q}$ is defined by $[1,2]$

$$
{ }_{p} F_{q}\left[\begin{array}{ccc}
\alpha_{1}, & \ldots, & \alpha_{p} ; \\
\beta_{1}, & \ldots, & \beta_{q} ;
\end{array}\right]=\sum_{n=0}^{\infty} \frac{\left(\alpha_{1}\right)_{n} \cdots\left(\alpha_{p}\right)_{n}}{\left(\beta_{1}\right)_{n} \cdots\left(\beta_{q}\right)_{n}} \frac{z^{n}}{n !},
$$

where $(a)_{n}$ is the Pochhammer symbol (or the shifted or raised factorial, since $\left.(1)_{n}=n !\right)$ defined (for $a \in \mathbb{C}$ ) by

$$
\begin{aligned}
(a)_{n} & = \begin{cases}1, & n=0, \\
a(a+1) \cdots(a+n-1), & n \in \mathbb{N}=\{1,2, \ldots\}\end{cases} \\
& =\frac{\Gamma(a+n)}{\Gamma(a)}, \quad a \in \mathbb{C} \backslash \mathbb{Z}_{0}^{-}
\end{aligned}
$$

and $\mathbb{Z}_{0}^{-}$denotes the set of non-positive integers, $\mathbb{C}$ the set of complex numbers, and $\Gamma(a)$ is the familiar gamma function. Here, $p$ and $q$ are positive integers or zero (interpreting an empty product as unity), and we assume for simplicity that the variable $z$, the numerator parameters $\alpha_{1}, \ldots, \alpha_{p}$ and the denominator parameters $\beta_{1}, \ldots, \beta_{q}$ take on complex values, provided that no zeros appear in the denominator of $(1)$, that is,

$$
\beta_{j} \in \mathbb{C} \backslash \mathbb{Z}_{0}^{-} ; \quad j=1, \ldots, q .
$$

@2013 Ibrahim et al.; licensee Springer. This is an Open Access article distributed under the terms of the Creative Commons Attribution License (http://creativecommons.org/licenses/by/2.0), which permits unrestricted use, distribution, and reproduction in any medium, provided the original work is properly cited. 
For the detailed conditions of the convergence of series (1), we refer to [2]. It is not out of place to mention here that if one of the numerator parameters, say $a_{j}$, is a negative integer, then series (1) reduces to a polynomial in $z$ of degree $-a_{j}$.

It is interesting to mention here that whenever a generalized hypergeometric function reduces to a gamma function, the results are very important from the application point of view. Thus the classical summation theorems such as those of Gauss, Gauss second, Kummer and Bailey for the series ${ }_{2} F_{1}$, Watson, Dixon, Whipple and Saalschütz for the series ${ }_{3} F_{2}$ and others play an important role in the theory of hypergeometric and generalized hypergeometric series.

In a very interesting, popular and useful research paper, Bailey [3], by employing the above mentioned classical summation theorems, obtained a large number of results involving products of generalized hypergeometric series as well as quadratic and cubic transformations. Several other results were also given by Gauss and Kummer.

Evidently, if the product of two generalized hypergeometric series can be expressed as another generalized hypergeometric series with argument $x$, the coefficients of $x^{n}$ in the product must be expressible in terms of gamma functions.

In our present investigation, we are interested in the following quadratic transformation due to Kummer [4]:

$$
(1-x)^{-2 a}{ }_{2} F_{1}\left[\begin{array}{lll}
2 a, & b ; & \\
2 b ; & & -\frac{2 x}{1-x}
\end{array}\right]={ }_{2} F_{1}\left[\begin{array}{lll}
a, & a+\frac{1}{2} ; & \\
b+\frac{1}{2} ; & & x^{2}
\end{array}\right] .
$$

This result was independently rediscovered by Ramanujan [5, Entry 2, p.49].

Using (4) and employing the so-called beta integral method, recently Krattenthaler and Rao [6, Eq. (3.4), p.164] obtained the following interesting identity:

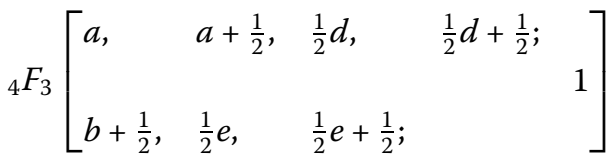

$$
\begin{aligned}
& =\frac{\Gamma(e) \Gamma(e-2 a-d)}{\Gamma(e-2 a) \Gamma(e-d)}{ }_{3} F_{2}\left[\begin{array}{llll}
2 a, & b, & d ; & \\
2 b, & 1+2 a+d-e ; &
\end{array}\right]
\end{aligned}
$$

provided $2 a$ or $d$ is a nonpositive integer.

Very recently, Kim et al. [7] have obtained a generalization of Kummer's quadratic transformation formula (4) in the form

$$
\begin{aligned}
(1-x)^{-2 a}{ }_{2} F_{1}\left[\begin{array}{lll}
2 a, & b ; & -\frac{2 x}{1-x} \\
2 b+j ; & &
\end{array}\right] \\
=\frac{\Gamma(b) \Gamma(1-b)}{\Gamma\left(b+\frac{1}{2} j+\frac{1}{2}|j|\right) \Gamma\left(1-b-\left[\frac{j+1}{2}\right]\right)} \\
\quad \cdot \sum_{n=0}^{\infty} A_{j}(n) \frac{x^{2 n}}{n !} \frac{(a)_{n}\left(a+\frac{1}{2}\right)_{n}\left(b+\left[\frac{j+1}{2}\right]\right)_{n}}{\left(b+\frac{1}{2} j\right)_{n}\left(b+\frac{1}{2} j+\frac{1}{2}\right)_{n}}
\end{aligned}
$$


Table 1 The coefficients $A_{j}$ and $B_{j}$

\begin{tabular}{rll}
\hline $\boldsymbol{j}$ & $\boldsymbol{A}_{\boldsymbol{j}}(\boldsymbol{n})$ & $\boldsymbol{B}_{\boldsymbol{j}}(\boldsymbol{n})$ \\
\hline 5 & $-4(1-b-2 n)^{2}$ & $4(b+2 n)^{2}-2(1-b)(b+2 n)$ \\
& $+2(1-b)(1-b-2 n)+(1-b)^{2}$ & $-(1-b)^{2}+34(b+2 n)+b+61$ \\
& $+22(1-b-2 n)+13 b-33$ & \\
4 & $2(b+1+2 n)(b+3+2 n)$ & $4(b+3+2 n)$ \\
& $-b(b+3)$ & \\
3 & $b+2+4 n$ & $-(3 b+6+4 n)$ \\
2 & $-(b+1+2 n)$ & -2 \\
1 & -1 & 1 \\
0 & 1 & 0 \\
-1 & 1 & 1 \\
-2 & $1-b-2 n$ & 2 \\
-3 & $1-b-4 n$ & $3-3 b-4 n$ \\
-4 & $2(1-b-2 n)(3-b-2 n)$ & $4(1-b-2 n)$ \\
& $-(1-b)(4-b)$ & $4(b+2 n)^{2}-2(1-b)(b+2 n)$ \\
-5 & $4(1-b-2 n)^{2}$ & $-(1-b)^{2}-16(b+2 n)+b-1$ \\
& $-2(1-b)(1-b-2 n)-(1-b)^{2}$ & \\
& $+8(1-b-2 n)+7 b-7$ & \\
& &
\end{tabular}

$$
\begin{aligned}
& +\frac{2 a}{(2 b+j)} \frac{\Gamma(-b) \Gamma(1+b)}{\Gamma\left(-b-\left[\frac{j}{2}\right]\right) \Gamma\left(b+\frac{1}{2} j+\frac{1}{2}|j|\right)} \\
& \cdot \sum_{n=0}^{\infty} B_{j}(n) \frac{x^{2 n+1}}{n !} \frac{\left(a+\frac{1}{2}\right)_{n}(a+1)_{n}\left(b+1+\left[\frac{j}{2}\right]\right)_{n}}{\left(b+\frac{1}{2} j+\frac{1}{2}\right)_{n}\left(b+\frac{1}{2} j+1\right)_{n}}
\end{aligned}
$$

for $j=0, \pm 1, \pm 2, \pm 3, \pm 4, \pm 5$.

Here, as usual, $[x]$ denotes the greatest integer less than or equal to $x$ and its modulus is denoted by $|x|$. The coefficients $A_{j}$ and $B_{j}$ are given in Table 1 .

Here, in this paper, we show how one can easily obtain eleven interesting hypergeometric identities including the Krattenthaler-Rao result (5) in the form of a single unified result by employing the beta integral method developed by Krattenthaler and Rao [6]. The results are derived with the help of generalization (6) of Kummer's formula (4). Several interesting special cases of our main result including (5) are also explicitly demonstrated.

The results presented in this paper are simple, interesting, easily established and (potentially) useful.

\section{Main result}

Our eleven main identities are given here in the form of a single unified result asserted in the following theorem.

Theorem 1 For $2 a$ or $d$ to be a non-positive integer, the following generalization of Krattenthaler-Rao formula (5) holds true:

$$
\begin{gathered}
\frac{\Gamma(e) \Gamma(e-2 a-d)}{\Gamma(e-2 a) \Gamma(e-d)}{ }_{3} F_{2}\left[\begin{array}{lll}
2 a, & b, & d ; \\
2 b+j, & 1+2 a+d-e ;
\end{array}\right] \\
=\frac{\Gamma(b) \Gamma(1-b)}{\Gamma\left(b+\frac{1}{2} j+\frac{1}{2}|j|\right) \Gamma\left(1-b-\left[\frac{j+1}{2}\right]\right)} \\
\cdot \sum_{n=0}^{\infty} \frac{A_{j}}{n !} \frac{(a)_{n}\left(a+\frac{1}{2}\right)_{n}\left(b+\left[\frac{j+1}{2}\right]\right)_{n}}{\left(b+\frac{1}{2} j\right)_{n}\left(b+\frac{1}{2} j+\frac{1}{2}\right)_{n}} \frac{\left(\frac{1}{2} d\right)_{n}\left(\frac{1}{2} d+\frac{1}{2}\right)_{n}}{\left(\frac{1}{2} e\right)_{n}\left(\frac{1}{2} e+\frac{1}{2}\right)_{n}}
\end{gathered}
$$




$$
\begin{aligned}
& +\frac{2 a}{(2 b+j)}\left(\frac{d}{e}\right) \frac{\Gamma(-b) \Gamma(1+b)}{\Gamma\left(b+\frac{1}{2} j+\frac{1}{2}|j|\right) \Gamma\left(-b-\left[\frac{j}{2}\right]\right)} \\
& \cdot \sum_{n=0}^{\infty} \frac{B_{j}}{n !} \frac{\left(a+\frac{1}{2}\right)_{n}(a+1)_{n}\left(b+1+\left[\frac{j}{2}\right]\right)_{n}}{\left(b+\frac{1}{2} j+\frac{1}{2}\right)_{n}\left(b+\frac{1}{2} j+1\right)_{n}} \frac{1}{\left.\left(\frac{1}{2} e+\frac{1}{2}\right)_{n}\left(\frac{1}{2} d+1\right)_{n} e+1\right)_{n}}
\end{aligned}
$$

for $j=0, \pm 1, \pm 2, \pm 3, \pm 4, \pm 5$. The coefficients $A_{j}$ and $B_{j}$ are the same as given in Table 1 .

Proof Let us assume that $2 a$ is a non-positive integer, say $2 a=-m$, where $m$ is a positive integer. Now, multiplying the left-hand side of (6) by $x^{d-1}(1-x)^{e-d-1}$, where we suppose temporarily that $\operatorname{Re}(e)>\operatorname{Re}(d)>0$, and integrating the resulting equation with respect to $x$ from 0 to 1 , expressing the involved ${ }_{2} F_{1}$ as a series and change the order of integration and summation, which is easily seen to be justified due to the uniform convergence of the involved series, and denoting it by $S_{1}$, we have

$$
S_{1}=\sum_{n=0}^{\infty} \frac{(2 a)_{n}(b)_{n}(-2)^{n}}{(2 b+j)_{n} n !} \int_{0}^{1} x^{d+n-1}(1-x)^{e-d-2 a-n-1} d x
$$

Evaluating the beta-integral and using the identity

$$
\Gamma(\alpha-n)=\frac{(-1)^{n} \Gamma(\alpha)}{(1-\alpha)_{n}}
$$

we have, after some algebra,

$$
S_{1}=\frac{\Gamma(d) \Gamma(e-d-2 a)}{\Gamma(e-2 a)} \sum_{n=0}^{\infty} \frac{(2 a)_{n}(b)_{n}(d)_{n}}{(2 b+j)_{n}(1+2 a+d-e)_{n}} \frac{2^{n}}{n !} .
$$

Summing up the series, we have

$$
S_{1}=\frac{\Gamma(d) \Gamma(e-d-2 a)}{\Gamma(e-2 a)}{ }_{3} F_{2}\left[\begin{array}{lll}
2 a, & b, & d ; \\
2 b+j, & 1+2 a+d-e ; & 2
\end{array}\right] .
$$

Also, multiplying the right-hand side of (6) by $x^{d-1}(1-x)^{e-d-1}$, and proceeding similarly as above and denoting it by $S_{2}$, we get after some simplification

$$
\begin{aligned}
S_{2}= & \frac{\Gamma(b) \Gamma(1-b)}{\Gamma\left(b+\frac{1}{2} j+\frac{1}{2}|j|\right) \Gamma\left(1-b-\left[\frac{j+1}{2}\right]\right)} \\
& \cdot \sum_{n=0}^{\infty} \frac{A_{j}}{n !} \frac{(a)_{n}\left(a+\frac{1}{2}\right)_{n}\left(b+\left[\frac{j+1}{2}\right]\right)_{n}}{\left(b+\frac{1}{2} j\right)_{n}\left(b+\frac{1}{2} j+\frac{1}{2}\right)_{n}} \int_{0}^{1} x^{d+2 n-1}(1-x)^{e-d-1} d x \\
& +\frac{2 a}{(2 b+j)} \frac{\Gamma(-b) \Gamma(1+b)}{\Gamma\left(b+\frac{1}{2} j+\frac{1}{2}|j|\right) \Gamma\left(-b-\left[\frac{j}{2}\right]\right)} \\
& \cdot \sum_{n=0}^{\infty} \frac{B_{j}}{n !} \frac{\left(a+\frac{1}{2}\right)_{n}(a+1)_{n}\left(b+1+\left[\frac{j}{2}\right]\right)_{n}}{\left(b+\frac{1}{2} j+\frac{1}{2}\right)_{n}\left(b+\frac{1}{2} j+1\right)_{n}} \int_{0}^{1} x^{d+2 n}(1-x)^{e-d-1} d x .
\end{aligned}
$$


Evaluating the beta integrals and using Legendre's duplication formula

$$
\Gamma(2 z)=\frac{2^{2 z-1} \Gamma(z) \Gamma\left(z+\frac{1}{2}\right)}{\sqrt{\pi}},
$$

we have, after some simplification,

$$
\begin{aligned}
S_{2}= & \frac{\Gamma(d) \Gamma(e-d)}{\Gamma(e)}\left\{\frac{\Gamma(b) \Gamma(1-b)}{\Gamma\left(b+\frac{1}{2} j+\frac{1}{2}|j|\right) \Gamma\left(1-b-\left[\frac{j+1}{2}\right]\right)}\right. \\
& \cdot \sum_{n=0}^{\infty} \frac{A_{j}}{n !} \frac{(a)_{n}\left(a+\frac{1}{2}\right)_{n}\left(b+\left[\frac{j+1}{2}\right]\right)_{n}}{\left(b+\frac{1}{2} j\right)_{n}\left(b+\frac{1}{2} j+\frac{1}{2}\right)_{n}} \frac{\left(\frac{1}{2}\right)_{n}\left(\frac{1}{2} d+\frac{1}{2}\right)_{n}}{\left(\frac{1}{2} e\right)_{n}\left(\frac{1}{2} e+\frac{1}{2}\right)_{n}} \\
& +\frac{2 a}{(2 b+j)}\left(\frac{d}{e}\right) \frac{\Gamma(-b) \Gamma(1+b)}{\Gamma\left(b+\frac{1}{2} j+\frac{1}{2}|j|\right) \Gamma\left(-b-\left[\frac{j}{2}\right]\right)} \\
& \left.\cdot \sum_{n=0}^{\infty} \frac{B_{j}}{n !} \frac{\left(a+\frac{1}{2}\right)_{n}(a+1)_{n}\left(b+1+\left[\frac{j}{2}\right]\right)_{n}}{\left(b+\frac{1}{2} j+\frac{1}{2}\right)_{n}\left(b+\frac{1}{2} j+1\right)_{n}} \frac{\left(\frac{1}{2} d+\frac{1}{2}\right)_{n}\left(\frac{1}{2} d+1\right)_{n}}{\left(\frac{1}{2} e+\frac{1}{2}\right)_{n}\left(\frac{1}{2} e+1\right)_{n}}\right\} .
\end{aligned}
$$

Finally, equating (8) and (9), we get the desired result (7). This completes the proof of (7). The above restriction on the parameters $d$ and $e$ may now be removed by appeal to analytic continuation.

\section{Special cases}

Here we shall consider some of the very interesting special cases of our main result (7). Each of the following formulas holds true provided $2 a$ or $d$ must be a non-positive integer.

\section{Corollary 2}

$$
\begin{gathered}
\frac{\Gamma(e) \Gamma(e-2 a-d)}{\Gamma(e-2 a) \Gamma(e-d)}{ }_{3} F_{2}\left[\begin{array}{lll}
2 a, & b, & d ; \\
2 b, & 1+2 a+d-e ; &
\end{array}\right] \\
\quad={ }_{4} F_{3}\left[\begin{array}{llll}
a, & a+\frac{1}{2}, & \frac{1}{2} d, & \frac{1}{2} d+\frac{1}{2} ; \\
b+\frac{1}{2}, & \frac{1}{2} e, & \frac{1}{2} e+\frac{1}{2} ; & 1
\end{array}\right]
\end{gathered}
$$

Proof Setting $j=0$ in (7) and simplifying the resulting identity, we are led to formula (10).

\section{Corollary 3}

$$
\begin{aligned}
& \frac{\Gamma(e) \Gamma(e-2 a-d)}{\Gamma(e-2 a) \Gamma(e-d)}{ }_{3} F_{2}\left[\begin{array}{llll}
2 a, & b, & d ; & \\
2 b+1, & 1+2 a+d-e ; &
\end{array}\right] \\
& ={ }_{4} F_{3}\left[\begin{array}{lllll}
a, & a+\frac{1}{2}, & \frac{1}{2} d, & \frac{1}{2} d+\frac{1}{2} ; & \\
b+\frac{1}{2}, & \frac{1}{2} e, & \frac{1}{2} e+\frac{1}{2} ; & & 1
\end{array}\right]
\end{aligned}
$$




$$
+\frac{2 a d}{e(2 b+1)}{ }_{4} F_{3}\left[\begin{array}{llll}
a+\frac{1}{2}, & a+1, & \frac{1}{2} d+\frac{1}{2}, & \frac{1}{2} d+1 ; \\
b+\frac{3}{2}, & \frac{1}{2} e+\frac{1}{2}, & \frac{1}{2} e+1 ; &
\end{array}\right]
$$

Proof Setting $j=1$ in (7) and simplifying the resulting identity, we are led to formula (11).

\section{Corollary 4}

$$
\begin{aligned}
& \frac{\Gamma(e) \Gamma(e-2 a-d)}{\Gamma(e-2 a) \Gamma(e-d)}{ }_{3} F_{2}\left[\begin{array}{ccc}
2 a, & b, & d ; \\
2 b-1, & 1+2 a+d-e ; & 2
\end{array}\right] \\
& \quad={ }_{4} F_{3}\left[\begin{array}{llll}
a, & a+\frac{1}{2}, & \frac{1}{2} d, & \frac{1}{2} d+\frac{1}{2} ; \\
b-\frac{1}{2}, & \frac{1}{2} e, & \frac{1}{2} e+\frac{1}{2} ; & 1
\end{array}\right] \\
& \quad-\frac{2 a d}{e(2 b-1)}{ }_{4} F_{3}\left[\begin{array}{cccc}
a+\frac{1}{2}, & a+1, & \frac{1}{2} d+\frac{1}{2}, & \frac{1}{2} d+1 ; \\
b+\frac{1}{2}, & \frac{1}{2} e+\frac{1}{2}, & \frac{1}{2} e+1 ; &
\end{array}\right] .
\end{aligned}
$$

Proof Setting $j=-1$ in (7) and simplifying the resulting identity, we are led to formula (12).

\section{Corollary 5}

$$
\begin{aligned}
& \frac{\Gamma(e) \Gamma(e-2 a-d)}{\Gamma(e-2 a) \Gamma(e-d)}{ }_{3} F_{2}\left[\begin{array}{llll}
2 a, & b, & d ; & \\
2 b+2, & 1+2 a+d-e ; &
\end{array}\right] \\
& ={ }_{5} F_{4}\left[\begin{array}{lllll}
a, & a+\frac{1}{2}, & \frac{1}{2} b+\frac{3}{2}, & \frac{1}{2} d, & \frac{1}{2} d+\frac{1}{2} ; \\
\frac{1}{2} b+\frac{1}{2}, & b+\frac{3}{2}, & \frac{1}{2} e, & \frac{1}{2} e+\frac{1}{2} ; &
\end{array}\right] \\
& +\frac{2 a d}{e(b+1)}{ }_{4} F_{3}\left[\begin{array}{llll}
a+\frac{1}{2}, & a+1, & \frac{1}{2} d+\frac{1}{2}, & \frac{1}{2} d+1 ; \\
b+\frac{3}{2}, & \frac{1}{2} e+\frac{1}{2}, & \frac{1}{2} e+1 ; &
\end{array}\right] .
\end{aligned}
$$

Proof Setting $j=2$ in (7) and simplifying the resulting identity, we are led to formula (13).

\section{Corollary 6}

$$
\begin{aligned}
& \frac{\Gamma(e) \Gamma(e-2 a-d)}{\Gamma(e-2 a) \Gamma(e-d)}{ }_{3} F_{2}\left[\begin{array}{llll}
2 a, & b, & d ; & \\
2 b-2, & 1+2 a+d-e ; &
\end{array}\right] \\
& ={ }_{5} F_{4}\left[\begin{array}{lllll}
a, & a+\frac{1}{2}, & \frac{1}{2} b+\frac{1}{2}, & \frac{1}{2} d, & \frac{1}{2} d+\frac{1}{2} ; \\
\frac{1}{2} b-\frac{1}{2}, & b-\frac{1}{2}, & \frac{1}{2} e, & \frac{1}{2} e+\frac{1}{2} ; & 1
\end{array}\right]
\end{aligned}
$$




$$
-\frac{2 a d}{e(b-1)}{ }_{4} F_{3}\left[\begin{array}{llll}
a+\frac{1}{2}, & a+1, & \frac{1}{2} d+\frac{1}{2}, & \frac{1}{2} d+1 ; \\
b-\frac{1}{2}, & \frac{1}{2} e+\frac{1}{2}, & \frac{1}{2} e+1 ; &
\end{array}\right] .
$$

Proof Setting $j=-2$ in (7) and simplifying the resulting identity, we are led to formula (14).

\section{Corollary 7}

$$
\begin{aligned}
& \frac{\Gamma(e) \Gamma(e-2 a-d)}{\Gamma(e-2 a) \Gamma(e-d)}{ }_{3} F_{2}\left[\begin{array}{llll}
2 a, & b, & d ; \\
2 b+3, & 1+2 a+d-e ; & 2
\end{array}\right] \\
& \quad={ }_{5} F_{4}\left[\begin{array}{lllll}
a, & a+\frac{1}{2}, & \frac{1}{4} b+\frac{3}{2}, & \frac{1}{2} d, & \frac{1}{2} d+\frac{1}{2} ; \\
\frac{1}{4} b+\frac{1}{2}, & b+\frac{3}{2}, & \frac{1}{2} e, & \frac{1}{2} e+\frac{1}{2} ; & 1
\end{array}\right] \\
& +\frac{6 a d}{e(2 b+3)}{ }_{5} F_{4}\left[\begin{array}{lllll}
a+\frac{1}{2}, & a+1, & \frac{3}{4} b+\frac{5}{2}, & \frac{1}{2} d+\frac{1}{2}, & \frac{1}{2} d+1 ; \\
\frac{3}{4} b+\frac{3}{2}, & b+\frac{5}{2}, & \frac{1}{2} e+\frac{1}{2}, & \frac{1}{2} e+1 ; & 1
\end{array}\right] .
\end{aligned}
$$

Proof Setting $j=3$ in (7) and simplifying the resulting identity, we are led to formula (15).

\section{Corollary 8}

$$
\begin{aligned}
& \frac{\Gamma(e) \Gamma(e-2 a-d)}{\Gamma(e-2 a) \Gamma(e-d)}{ }_{3} F_{2}\left[\begin{array}{cccc}
2 a, & b, & d ; \\
2 b-3, & 1+2 a+d-e ; & 2
\end{array}\right] \\
& \quad={ }_{5} F_{4}\left[\begin{array}{lllll}
a, & a+\frac{1}{2}, & \frac{1}{4} b+\frac{3}{4}, & \frac{1}{2} d, & \frac{1}{2} d+\frac{1}{2} ; \\
\frac{1}{4} b-\frac{1}{4}, & b-\frac{3}{2}, & \frac{1}{2} e, & \frac{1}{2} e+\frac{1}{2} ; & 1
\end{array}\right] \\
& -\frac{6 a d}{e(2 b-3)}{ }_{5} F_{4}\left[\begin{array}{ccccc}
a+1, & a+\frac{1}{2}, & \frac{3}{4} b+\frac{1}{4}, & \frac{1}{2} d+\frac{1}{2}, & \frac{1}{2} d+1 ; \\
\frac{3}{4} b-\frac{3}{4}, & b-\frac{1}{2}, & \frac{1}{2} e+\frac{1}{2}, & \frac{1}{2} e+1 ; & 1
\end{array}\right] .
\end{aligned}
$$

Proof Setting $j=-3$ in (7) and simplifying the resulting identity, we are led to formula (16).

Result (10) is the well-known result of Krattenthaler and Rao [6] and results (11) to (16) are closely related to it.

Remark 9 We conclude this section by mentioning that the series ${ }_{5} F_{4}$ appearing on the right-hand side of results (13) to (16) can also be written as a sum of two ${ }_{4} F_{3}$, and then we can obtain alternate forms of the results. 


\section{Competing interests}

The authors declare that they have no competing interests.

\section{Authors' contributions}

All authors contributed equally in this paper. They read and approved the final manuscript.

\section{Author details}

${ }^{1}$ Department of Mathematics, Faculty of Science, Jazan University, Jazan, Saudi Arabia. ${ }^{2}$ Department of Mathematics and Statistics - College of Science, Sultan Qaboos University, Alkhoud, P.O. Box 36, Muscat, 123, Oman. ${ }^{3}$ Permanent address: Department of Mathematics, Faculty of Science, Suez Canal University, Ismailia, Egypt. ${ }^{4}$ Department of Mathematics, School of Mathematical and Physical Sciences, Central University of Kerala, Riverside Transit Campus, Padennakkad P.O. Nileshwar, Kasaragod, Kerala 671 328, India.

\section{Acknowledgements}

The authors are thankful to the referee for making certain very useful suggestions. The work of this research paper was supported by the research grant (05/4/33) funded by Jazan University - Jazan, Saudi Arabia.

\section{Received: 12 August 2013 Accepted: 29 October 2013 Published: 25 Nov 2013}

\section{References}

1. Bailey, WN: Generalized Hypergeometric Series. Cambridge University Press, Cambridge (1964)

2. Rainville, ED: Special Functions. Chelsea Publishing, New York (1971)

3. Bailey, WN: Products of generalized hypergeometric series. Proc. Lond. Math. Soc. 28, 242-254 (1928)

4. Kummer, EE: Uber die hypergeometrische reihe $1+\frac{\alpha \cdot \beta}{1 \cdot \gamma} x+\frac{\alpha(\alpha+1) \cdot \beta(\beta+1)}{1 \cdot 2 \gamma(\gamma+1)} x^{2}+\cdots$. J. Reine Angew. Math. 15, 39-83 (1836)

5. Berndt, BC: Ramanujan's Notebooks, Part II. Springer, Berlin (1989)

6. Krattenthaler, C, Rao, KS: Automatic generation of hypergeometric identities by the beta integral method. J. Comput. Appl. Math. 160, 159-173 (2003)

7. Kim, YS, Rakha, MA, Rathie, AK: Generalizations of Kummer's second theorem with applications. Comput. Math. Math. Phys. 50, 387-402 (2010)

10.1186/1687-1847-2013-341

Cite this article as: Ibrahim et al.: On certain hypergeometric identities deducible by using the beta integral method. Advances in Difference Equations 2013, 2013:341

\section{Submit your manuscript to a SpringerOpen ${ }^{\odot}$ journal and benefit from:}

- Convenient online submission

- Rigorous peer review

- Immediate publication on acceptance

- Open access: articles freely available online

- High visibility within the field

- Retaining the copyright to your article 